\title{
Cannabidiol attenuates seizures and EEG abnormalities in Angelman syndrome model mice
}

\author{
Bin Gu, ${ }^{1,2}$ Manhua Zhu, ${ }^{3}$ Madison R. Glass, ${ }^{3}$ Marie Rougié, ${ }^{1}$ Viktoriya D. Nikolova, ${ }^{4,5}$ Sheryl S. Moy, ${ }^{4,5}$ Paul R. Carney, ${ }^{3,5,6,7}$ \\ and Benjamin D. Philpot,
}

DDepartment of Cell Biology and Physiology, ${ }^{2}$ Neuroscience Center, ${ }^{3}$ Neuroscience Curriculum, ${ }^{4}$ Department of Psychiatry, ${ }^{5}$ Carolina Institute for Developmental Disabilities, ${ }^{6}$ Department of Neurology, and ${ }^{7}$ Department of Pediatrics, University of North Carolina, Chapel Hill, North Carolina, USA.

\begin{abstract}
Angelman syndrome (AS) is a neurodevelopmental disorder characterized by intellectual disability, lack of speech, ataxia, EEG abnormalities, and epilepsy. Seizures in individuals with AS are common, debilitating, and often drug resistant. Thus, there is an unmet need for better treatment options. Cannabidiol (CBD), a major phytocannabinoid constituent of cannabis, has shown antiseizure activity and behavioral benefits in preclinical and clinical studies for some disorders associated with epilepsy, suggesting that the same could be true for AS. Here, we show that acute CBD $(100 \mathrm{mg} / \mathrm{kg})$ treatment attenuated hyperthermia- and acoustically induced seizures in a mouse model of AS. However, neither acute CBD nor a 2-week-long course of CBD administered immediately after a kindling protocol could halt the proepileptogenic plasticity observed in AS model mice. CBD had a dose-dependent sedative effect but did not have an impact on motor performance. CBD abrogated the enhanced intracortical local field potential power, including the delta and theta rhythms observed in AS model mice, indicating that CBD administration could also help normalize the EEC deficits observed in individuals with AS. We believe our results provide critical preclinical evidence supporting CBD treatment of seizures and alleviation of EEG abnormalities in AS and will thus help guide the rational development of CBD as a treatment for $A S$.
\end{abstract}

\section{Introduction}

Deletions or mutations of the maternally inherited copy of the $U B E 3 A$ gene cause Angelman syndrome (AS). Individuals with AS show developmental delay, motor dysfunction, minimal speech, highly penetrant EEG abnormalities, and seizures (1, 2). Epilepsy in AS is common (80\%-95\%), polymorphic, and often resistant to available antiepileptic drugs. The frequency, severity, and pharmacological intractability of the seizures exact a heavy toll on individuals with AS and their caregivers (3-6). AS model mice lacking a functional maternal copy of the orthologous Ube3a gene $\left(U_{b e} 3 a^{m-p^{+}}\right)$phenocopy many clinical aspects of AS, including seizure susceptibility, motor and behavioral impairments, as well as EEG abnormalities, thus offering a preclinical model for the development of new therapeutics (7-15).

Cannabidiol (CBD), a major phytocannabinoid constituent of cannabis, is gaining attention for its antiepileptic, anxiolytic, and antipsychotic properties (16). In 2018, the FDA approved CBD (Epidiolex) for the treatment of seizures associated with 2 rare and severe forms of epilepsy - Lennox-Gastaut syndrome and Dravet syndrome. Although the interest and off-label medical use of CBD has largely outpaced the preclinical and clinical research, CBD provides a viable treatment for several other neurological disorders associated with epilepsy (17-20). However, little is known about the potential antiepileptic and other benefits

Conflict of interest: The authors have declared that no conflict of interest exists. Copyright: @ 2019, American Society for Clinical Investigation.

Submitted: May 20, 2019; Accepted: September 3, 2019; Published: November 4, 2019 Reference information: J Clin Invest. 2019;129(12):5462-5467.

https://doi.org/10.1172/JCI130419. of CBD in AS. The potential medicinal effects of CBD hold promise for the simultaneous amelioration of behavioral deficits, EEG abnormalities, and seizures in AS (21).

In this study, we systematically tested the beneficial effects of CBD on seizures, motor deficits, and EEG abnormalities in mice that genetically model AS, with the expectation that this information will guide eventual clinical use. We report that acute treatment with CBD substantially attenuated audiogenic and hyperthermia-induced seizure severity and normalized delta rhythms in AS model mice. The anticonvulsant dose of CBD $(100 \mathrm{mg} / \mathrm{kg})$ caused mild sedation but had little effect on motor coordination or balance. While acute CBD could suppress seizure severity, CBD stopped short of being able to prevent the proepileptogenic plasticity observed in AS model mice. Our study provides a preclinical framework to better guide the rational development of $\mathrm{CBD}$ as either an adjunctive or monotherapy for AS.

\section{Results and Discussion}

As with individuals with AS, mice with maternal loss of Ube3a exhibit epileptic phenotypes. For example, AS mice on a 129 background have elevated seizure responses to acoustic stimuli $(9,10,14)$. We verified that AS model mice (on a 129 background) had more frequent audiogenic tonic convulsions than did WT littermates (Figure 1). We found that acute treatment with $\mathrm{CBD}$ at $100 \mathrm{mg} / \mathrm{kg}$ significantly reduced the frequency and severity of seizures triggered by acoustic stimuli (Figure 1). These results indicate strong dosedependent anticonvulsant effects of CBD in AS model mice.

We recently implemented the flurothyl kindling and retest paradigm in AS model mice (on a C57BL/6J background) and found that AS model mice responded to both initial (day 1) sei- 
A

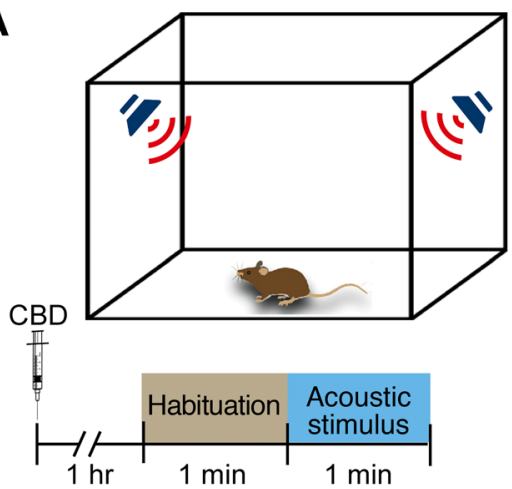

B

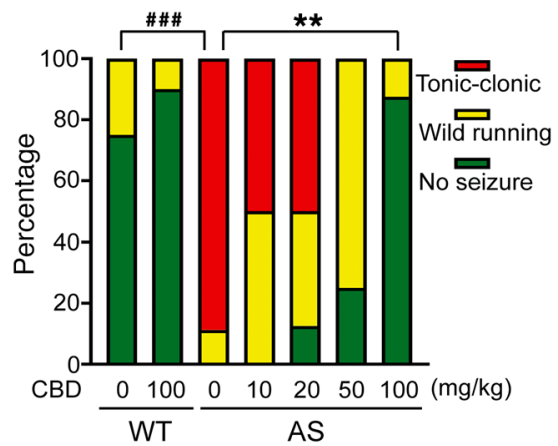

C

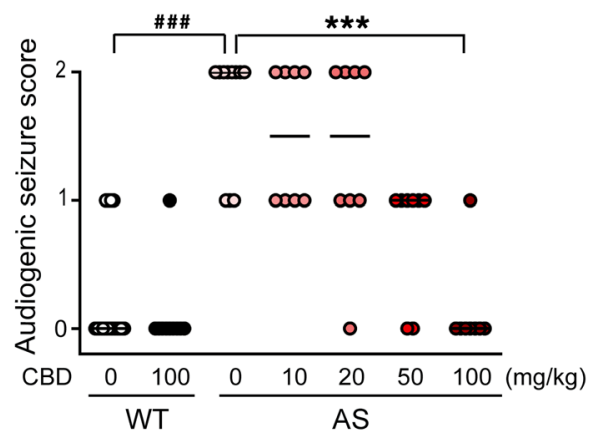

Figure 1. CBD attenuates wild running and tonic-clonic seizures induced by acoustic stimuli in AS model mice. (A) Schematic of the audiogenic seizure paradigm. (B) Treatment (i.p.) with CBD at $100 \mathrm{mg} / \mathrm{kg}$ significantly reduced audiogenic seizure frequency. $n=8-16$ mice/group. \#\#\#<0.001 compared with vehicle-treated WT mice; ${ }^{*} P<0.01$ compared with vehicle-treated AS mice; Fisher's exact test. (C) CBD at $100 \mathrm{mg} / \mathrm{kg}$ significantly reduced audiogenic seizure severity. Score 0 = no seizure response; score 1 = wild running and jumping; score 2 = tonic-clonic clonus. Data represent the median for individual mice. $n=$ 8-16 mice/group. ${ }^{\# \# P} P 0.001$ compared with vehicle-treated WT mice; ${ }^{* * *} P<0.001$ compared with vehicle-treated AS mice; Kruskal-Wallis test with Dunn's multiple comparisons test.

zure induction and kindling (day 1 to day 8) in a manner similar to that seen in WT mice, but the AS model mice displayed a markedly increased sensitivity to flurothyl-induced myoclonic and generalized seizures measured 1 month later at retest (day 36) (8). Elevation of core body temperature also triggered convulsions in kindled AS but not WT mice, resembling the clinical observation that individuals with AS are susceptible to febrile seizures with moderate increases in body temperature $(3,4,8)$. To test whether the anticonvulsant effects of CBD are generalizable across different seizure induction paradigms, we treated flurothyl-kindled mice acutely with CBD $(100 \mathrm{mg} / \mathrm{kg}) 1$ hour prior to the flurothyl or hyperthermia stimuli (Figure 2A). Surprisingly, the acute administration of CBD had little effect on the flurothyl-induced myoclonic or generalized seizure threshold (Figure 2, B and C) or the body temperature for the onset of hyperthermia-induced generalized seizure in kindled AS mice (Figure 2D). However, CBD significantly attenuated the severity and duration of hyperthermia-induced generalized seizures in kindled AS mice (Figure 2, E and F), once again supporting a role for $\mathrm{CBD}$ in attenuating seizure severity.

Figure 2. CBD reduces hyperthermia-induced generalized seizure duration and severity in the kindled AS model mice. (A) Schematic of flurothyl kindling and retest followed by hyperthermia-induced seizure. (B) Myoclonic and (C) generalized seizure threshold upon flurothyl retesting of kindled WT or AS model mice treated with either vehicle (Veh) or CBD (100 mg/ $\mathrm{kg}$, i.p.) 1 hour prior to the retest. Data represent the mean \pm SEM. $n=4-7$ mice/group. ${ }^{\# \#} P<0.01$ and $\# \# P<0.001$ compared with vehicle-treated WT mice; 2-way ANOVA with Tukey's post hoc test. Hyperthermia-induced generalized seizure (D) onset body temperature, (E) duration, and (F) maximum seizure score of kindled AS mice treated with vehicle or $\mathrm{CBD}(100 \mathrm{mg} / \mathrm{kg}$, i.p.). Data represent individual mice and the mean \pm SEM (D and $\mathbf{E}$ ) or median (F). $n=5-7$ mice/ group. ${ }^{*} P<0.05$ compared with vehicle-treated AS mice; unpaired, 2-tailed $t$ test ( $\mathbf{D}$ and $\mathbf{E}$ ) and Mann-Whitney $U$ test (F). 

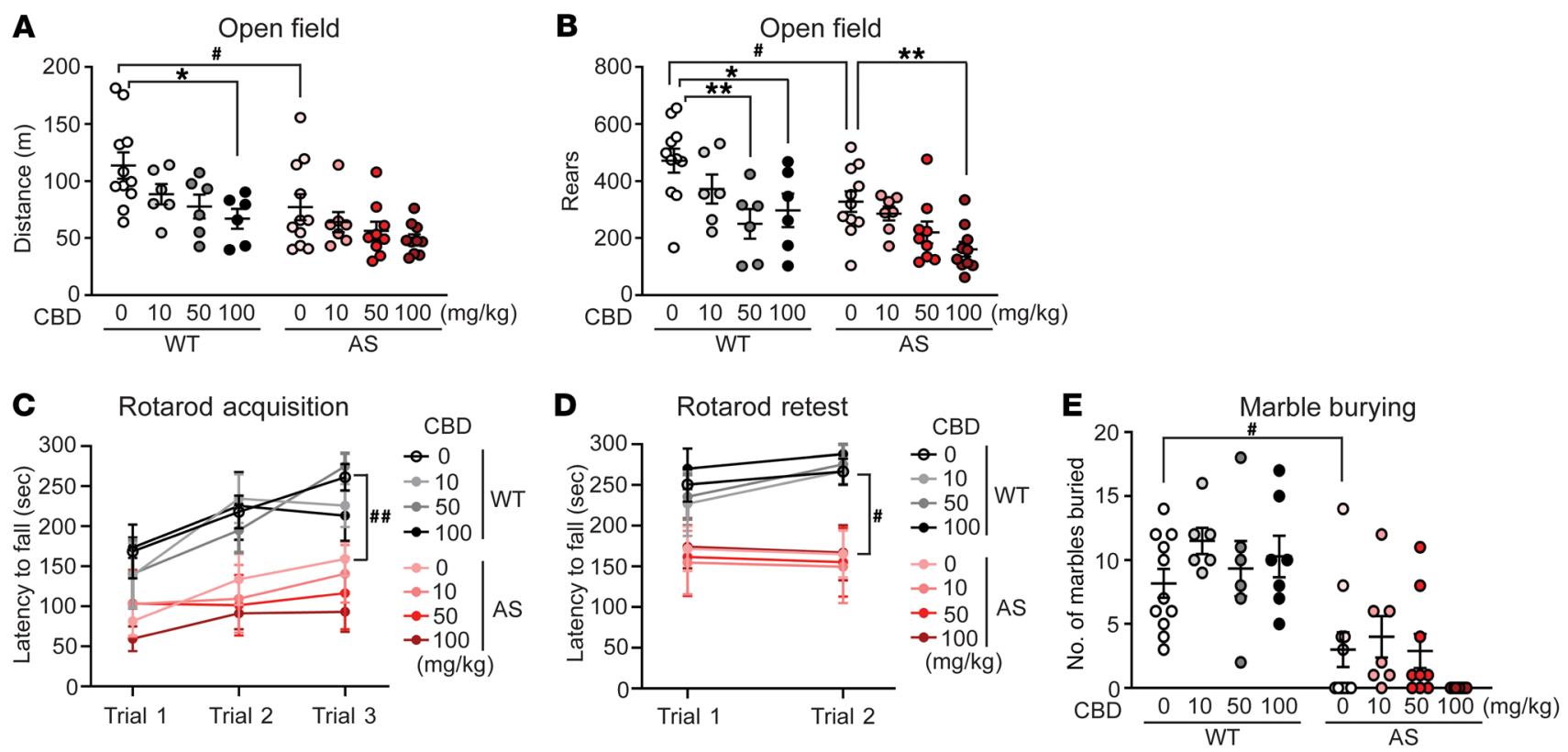

Figure 3. CBD has moderate sedative effects in AS model mice. (A and B) WT and AS model mice were subjected to an open-field test to assess (A) horizontal (distance traveled) and (B) vertical (rears) movement 1 hour after injection of vehicle or CBD. (C and D) Latency to fall from an accelerating rotarod in each trial of (C) acquisition and (D) retest session. Vehicle or CBD was injected i.p. 1 hour prior to the acquisition session. (E) Number of marbles buried by WT and AS model mice treated i.p. with vehicle or CBD 1 hour prior to the test. Note that none of the $10 \mathrm{AS}$ model mice treated with $100 \mathrm{mg} / \mathrm{kg}$ CBD buried a marble during the test. Data represent the mean \pm SEM. $n=6-11$ mice/group. ${ }^{\#} P<0.05$ and ${ }^{\# \#} P<0.01$ compared with vehicle-treated WT mice; ${ }^{*} P<0.05$ and ${ }^{* *} P<0.01$ compared with vehicle-treated AS mice; 2-way ANOVA with Tukey's post hoc test.

Aside from the promising anticonvulsant effect of CBD, little is known about the possible antiepileptogenic effect of CBD. This can be studied in a flurothyl kindling and retest model, as AS mice show seizure susceptibility similar to that of WT mice across an initial 8 days of flurothyl kindling but then exhibit a proepileptogenic plasticity during the subsequent month-long incubation period that renders them highly susceptible to seizures compared with WT mice (8). To test whether CBD can exert an antiepileptogenic effect, we initiated a 2-week CBD treatment (100 mg/kg, i.p. once per day) immediately after completion of flurothyl kindling, followed by a 2-week drug washout prior to the flurothyl retest (Supplemental Figure 1A; supplemental material available online with this article; https://doi.org/10.1172/JCI130419DS1). Consistent with our previous findings (8), vehicle-treated AS mice exhibited enhanced myoclonic and generalized seizure susceptibilities at flurothyl retest compared with WT mice (Supplemental Figure 1, B and C). Moreover, the post-kindling CBD treatments failed to attenuate the enhanced myoclonic or generalized seizure susceptibility measured in AS model mice at flurothyl retest (Supplemental Figure 1, D and E), suggesting that 2-week-long CBD treatments (at $100 \mathrm{mg} / \mathrm{kg}$ ) cannot prevent the proepileptogenic plasticity that occurs following kindling in AS mice. Importantly, previous studies demonstrated that there are critical windows of therapeutic interventions for preventing many AS phenotypes, including proepileptogenic plasticity in mice $(8,14)$. Thus, there is a future need to examine possible age-dependent consequences of $\mathrm{CBD}$ administration.

Motor and behavioral impairments are common in children with AS, significantly affect their daily lives, and increase the burden of their caregivers. No effective drug treatments are available. AS model mice exhibit behavioral and motor deficits $(7,9,15,22)$, some of which resemble those observed in individuals with AS. To explore possible behavioral benefits of $\mathrm{CBD}$, we treated AS model mice and WT controls with various doses of CBD 1 hour prior to behavioral testing. Similar to prior observations $(7,9,15,22)$, we found that vehicle-treated AS model mice exhibit impaired locomotor activity, poor motor coordination, and reduced marble-burying behavior (Figure 3). Open-field activity was reduced in AS model mice, and CBD had a dose-dependent sedative effect in both WT and AS mice (Figure 3, A and B). Consistent with previous findings in rats (23), CBD did not have a major impact on motor skill learning or memory, regardless of genotype, as measured in rotarod acquisition and retesting (Figure 3, C and D). Notably, CBD at $100 \mathrm{mg} / \mathrm{kg}$ exaggerated the marble-burying deficits of AS model mice (Figure $3 \mathrm{E}$ ), which might be a consequence of its sedative effects.

Individuals with AS have higher EEG power across all frequencies compared with neurotypical controls, with the largest difference manifested in a prominent peak in the delta frequency range (13, 24-26). Electrophysiological recordings from freely roaming AS model mice indicate strain- and region-dependent quantitative differences in EEG power spectrum analysis, with the most robust elevations of delta and theta power found in the cortex of AS mice on a C57BL/6J background, regardless of light cycle (7). Here, we monitored and quantified the cortical local field potential (LFP) of freely roaming AS and WT mice (on C57BL/6J background) after 2 weeks of vehicle or CBD treatment (Figure 4). Consistent with previous findings (7), AS model mice exhibited enhanced electrophysiological power compared with WT mice, particularly with regard to delta $(1-4 \mathrm{~Hz})$ and theta $(5-8 \mathrm{~Hz})$ activity. CBD treatments had little effect on LFP power in WT mice, whereas the treatment significantly lowered total LFP power, including 

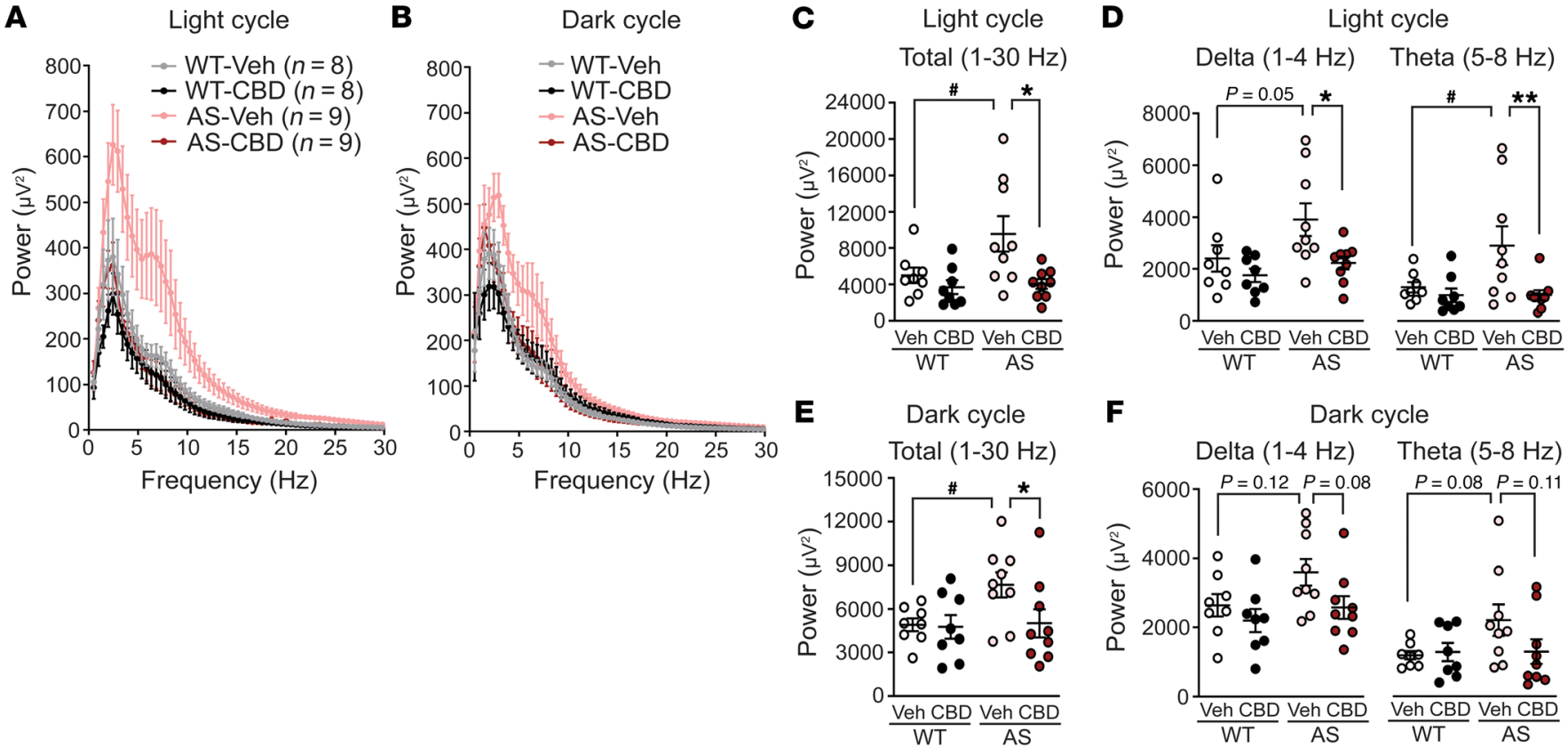

Figure 4. Two weeks of CBD administration normalizes LFP in AS model mice. (A and B) Power analyses of cortical LFP during the (A) light or (B) dark cycle of WT and AS model mice treated with vehicle (AS-Veh, WT-Veh) or CBD (AS-CBD, WT-CBD) $(100 \mathrm{mg} / \mathrm{kg}$, once daily, i.p.). (C-F) LFP power analyses of $(\mathbf{C}$ and $\mathbf{E})$ total $(1-30 \mathrm{~Hz})$ as well as $(\mathbf{D}$ and $\mathbf{F})$ delta $(1-4 \mathrm{~Hz})$ and theta $(5-8 \mathrm{~Hz})$ frequency bands during the (C and $\mathbf{D})$ light or $(\mathbf{E}$ and $\mathbf{F})$ dark cycle of WT and AS model mice treated with vehicle or CBD $\left(100 \mathrm{mg} / \mathrm{kg}\right.$, once daily, i.p.). Data represent the mean \pm SEM. $n=8-9$ mice/group. ${ }^{\#} P<0.05$ compared with vehicle-treated WT mice; ${ }^{*} P<0.05$ and ${ }^{* *} P<0.01$ compared with vehicle-treated AS mice; 2 -way ANOVA with Tukey's post hoc test.

both delta and theta activity, in AS model mice, normalizing levels to those observed in WT mice (Figure 4). All mice showed similar weight gain over the 2-week period, regardless of genotype or treatment (Supplemental Figure 2).

Our data are the first to our knowledge to show that CBD can reduce both acoustically and hyperthermia-induced seizure severity in AS model mice. As such, our findings suggest that CBD might attenuate seizures in individuals with AS, which expands the therapeutic spectrum of the antiepileptic effects of CBD (17, 19, 23, 27-32). The fact that neither acute nor chronic post-kindling CBD treatment affected the flurothyl- or hyperthermiainduced seizure threshold in AS model mice suggests that further evaluation of $\mathrm{CBD}$ using additional models of epilepsy is required to reveal the full antiepileptic potential of CBD in AS treatment. The context-dependent antiepileptic effects of CBD suggest that different seizure models can engage distinct mechanisms for seizure initiation and propagation. And, relevant to human use, such a finding indicates that CBD might be beneficial for some types of seizures and not others, depending on the circuits engaged and/or at what stage of epilepsy intervention begins.

CBD shows behavioral benefits in animal models of motor, social, and cognitive impairments (33-37). However, the behavioral benefits of CBD in animal models often exhibit an inverted U-shaped dose-response curve, with higher doses $(>20 \mathrm{mg} / \mathrm{kg}$ ) being ineffective and sedative and lower doses sometimes effective $(38,39)$. This conundrum for designing CBD dosing that both controls seizures and improves behavior was also found in studies of Dravet syndrome model mice (40). The same may be true for AS, as the effective concentration (e.g., $100 \mathrm{mg} / \mathrm{kg}$ ) at which we observed anticonvulsant effects also produced sedative effects. Notably, the sedative effects in mice at higher doses of CBD preclude meaningful interpretation of many movement-dependent behavioral paradigms (e.g., the 3-chamber sociability test, fear conditioning, and the Morris water maze) and may also explain the reduced marble-burying behavior, which can be associated with locomotor activity (41). However, although AS model mice recapitulate many AS-like behaviors, activity is not one of them, as these mice are hypoactive, whereas individuals with AS are often hyperactive (42). Thus, although anticonvulsant doses of CBD (i.e., $100 \mathrm{mg} / \mathrm{kg}$ ) are mildly sedative in AS model mice, it is conceivable that analogous CBD dosing could alleviate the hypermotor behavior often observed in individuals with AS.

EEG power is broadly increased in children with AS relative to age-matched neurotypical controls, and this is recapitulated under certain conditions in AS model mice relative to WT littermates $(7,24)$. Within the elevated EEG spectrum, enhanced delta rhythm is a particularly reliable biomarker for AS (13). Here, we found that 2 weeks of CBD administration reduced wide spectral cortical electrophysiological activity and normalized the delta (1-4 $\mathrm{Hz})$ and theta $(5-8 \mathrm{~Hz})$ activity in AS model mice. This suggests that CBD can suppress pathological EEG signatures in AS model mice, and perhaps in individuals with AS.

Although we experimentally controlled for our choice of CBD solvents and delivery routes, future experiments should more fully explore different administration routes and the potential effects of the ethanol and cremophor solvents. For example, administration of ethanol has been shown to interact with GABAergic transmission (43), and this could potentially influence our results. More- 
over, pharmacokinetic studies suggest that the half-life of CBD in rodents is generally shorter than that in humans $(44,45)$, therefore, it is difficult to make a precise comparison of the $100 \mathrm{mg} /$ $\mathrm{kg}$ effective i.p. dose in mice with the $20 \mathrm{mg} / \mathrm{kg}$ oral dose used in recent clinical trials $(27,32)$. Although thorough clinical studies are needed, we believe the present study lends critical preclinical support for the use of CBD to treat seizures, along with behavioral and EEG abnormalities in AS, and expands the potential beneficial spectrum of CBD treatment.

\section{Methods}

Detailed experimental methods are included in the supplemental materials. Male and female mice were used for experiments at equal genotypic ratios. Synthetic CBD $(99.2 \% \pm 0.18 \%$ purity) was provided by RTI International (log no. 3857-52-1). Data are presented as the mean \pm SEM. Unless otherwise noted, an unpaired, 2-tailed $t$ test was used for single comparisons, and a 2-way ANOVA was used for multiple comparisons. A $P$ value of less than 0.05 was considered significant.
Study approval. All animal procedures followed NIH guidelines and were approved by the IACUC of the University of North Carolina.

\section{Author contributions}

BG, MZ, and MRG performed experiments. BG, MZ, and MRG analyzed the data. MR managed the mouse colony and genotyping. BG, VDN, SSM, PRC, and BDP designed and coordinated the studies. BG, PRC, and BDP wrote the manuscript.

\section{Acknowledgments}

This work was supported by the Angelman Syndrome Foundation (to PRC and BDP); NIH grant R01HD093771 (to BDP); and a grant from the National Institute of Child Health and Human Development (NICHD) U54 Intellectual and Developmental Disabilities Research Center (HD079124, to SSM).

Address correspondence to: Ben Philpot, University of North Carolina, 116 Manning Drive, CB 7545, Chapel Hill, North Carolina 27599, USA. Phone: 919.966.0025; Email: bphilpot@med.unc.edu.
1. Buiting K, Williams C, Horsthemke B. Angelman syndrome - insights into a rare neurogenetic disorder. Nat Rev Neurol. 2016;12(10):584-593.

2. Williams CA, Driscoll DJ, Dagli AI. Clinical and genetic aspects of Angelman syndrome. Genet Med. 2010;12(7):385-395.

3. Bakke KA, Howlin P, Retterstøl L, Kanavin ØJ, Heiberg A, Nærland T. Effect of epilepsy on autism symptoms in Angelman syndrome. Mol Autism. 2018;9:2.

4. Fiumara A, Pittalà A, Cocuzza M, Sorge G. Epilepsy in patients with Angelman syndrome. Ital J Pediatr. 2010;36:31.

5. Laan LA, et al. Evolution of epilepsy and EEG findings in Angelman syndrome. Epilepsia. 1997;38(2):195-199.

6. Thibert RL, et al. Epilepsy in Angelman syndrome: a questionnaire-based assessment of the natural history and current treatment options. Epilepsia. 2009;50(11):2369-2376.

7. Born HA, et al. Strain-dependence of the Angelman syndrome phenotypes in Ube3a maternal deficiency mice. Sci Rep. 2017;7(1):8451.

8. Gu B, et al. Ube3a reinstatement mitigates epileptogenesis in Angelman syndrome model mice. JClin Invest. 2019;129(1):163-168.

9. Jiang YH, et al. Mutation of the Angelman ubiquitin ligase in mice causes increased cytoplasmic p53 and deficits of contextual learning and longterm potentiation. Neuron. 1998;21(4):799-811.

10. Judson MC, et al. GABAergic Neuron-specific loss of Ube3a causes Angelman syndrome-like EEG abnormalities and enhances seizure susceptibility. Neuron. 2016;90(1):56-69.

11. Mandel-Brehm C, Salogiannis J, Dhamne SC, Rotenberg A, Greenberg ME. Seizure-like activity in a juvenile Angelman syndrome mouse model is attenuated by reducing Arc expression. Proc Natl Acad Sci USA. 2015;112(16):5129-5134.

12. Miura K, et al. Neurobehavioral and electroencephalographic abnormalities in Ube3a maternal-deficient mice. Neurobiol Dis. 2002;9(2):149-159.

13. Sidorov MS, et al. Delta rhythmicity is a reliable EEG biomarker in Angelman syndrome: a parallel mouse and human analysis. JNeurodev Disord. 2017;9:17.

14. Silva-Santos S, et al. Ube3a reinstatement identifies distinct developmental windows in a murine Angelman syndrome model. J Clin Invest. 2015;125(5):2069-2076.

15. Sonzogni M, et al. A behavioral test battery for mouse models of Angelman syndrome: a powerful tool for testing drugs and novel Ube3a mutants. Mol Autism. 2018;9:47.

16. Izzo AA, Borrelli F, Capasso R, Di Marzo V, Mechoulam R. Non-psychotropic plant cannabinoids: new therapeutic opportunities from an ancient herb. Trends Pharmacol Sci. 2009;30(10):515-527.

17. Devinsky O, et al. Open-label use of highly purified CBD (Epidiolex) in patients with CDKL5 deficiency disorder and Aicardi, Dup15q, and Doose syndromes. Epilepsy Behav. 2018;86:131-137.

18. Hess EJ, et al. Cannabidiol as a new treatment for drug-resistant epilepsy in tuberous sclerosis complex. Epilepsia. 2016;57(10):1617-1624.

19. Devinsky O, et al. Cannabidiol in patients with treatment-resistant epilepsy: an open-label interventional trial. Lancet Neurol. 2016;15(3):270-278.

20. Gofshteyn JS, et al. Cannabidiol as a potential treatment for febrile infection-related epilepsy syndrome (FIRES) in the acute and chronic phases. J Child Neurol. 2017;32(1):35-40.

21. Ibeas Bih C, Chen T, Nunn AV, Bazelot M, Dallas M, Whalley BJ. Molecular targets of cannabidiol in neurological disorders. Neurotherapeutics. 2015;12(4):699-730.

22. Huang HS, et al. Behavioral deficits in an Angelman syndrome model: effects of genetic background and age. Behav Brain Res. 2013;243:79-90.

23. Jones NA, et al. Cannabidiol exerts anti-convulsant effects in animal models of temporal lobe and partial seizures. Seizure. 2012;21(5):344-352.

24. Frohlich J, et al. Electrophysiological phenotype in Angelman syndrome differs between genotypes. Biol Psychiatry. 2019;85(9):752-759.

25 . Valente KD, et al. Angelman syndrome: difficul- ties in EEG pattern recognition and possible misinterpretations. Epilepsia. 2003;44(8):1051-1063.

26. Vendrame M, et al. Analysis of EEG patterns and genotypes in patients with Angelman syndrome. Epilepsy Behav. 2012;23(3):261-265.

27. Devinsky O, et al. Trial of cannabidiol for drug-resistant seizures in the Dravet syndrome. N Engl JMed. 2017;376(21):2011-2020.

28. Devinsky O, et al. Long-term cannabidiol treatment in patients with Dravet syndrome: An open-label extension trial. Epilepsia. 2019;60(2):294-302.

29. Filloux FM. Cannabinoids for pediatric epilepsy? Up in smoke or real science? Transl Pediatr. 2015;4(4):271-282.

30. Friedman D, Devinsky O. Cannabinoids in the treatment of epilepsy. N Engl JMed. 2015;373(11):1048-1058.

31. Rosenberg EC, Tsien RW, Whalley BJ, Devinsky O. Cannabinoids and epilepsy. Neurotherapeutics. 2015;12(4):747-768.

32. Devinsky O, et al. Effect of cannabidiol on drop seizures in the Lennox-Gastaut syndrome. $\mathrm{NEngl}$ JMed. 2018;378(20):1888-1897.

33. Almeida V, et al. Cannabidiol exhibits anxiolytic but not antipsychotic property evaluated in the social interaction test. Prog Neuropsychopharmacol Biol Psychiatry. 2013;41:30-35.

34. Campos AC, Fogaça MV, Sonego AB, Guimarães FS. Cannabidiol, neuroprotection and neuropsychiatric disorders. Pharmacol Res. 2016;112:119-127.

35. Gururajan A, Taylor DA, Malone DT. Cannabidiol and clozapine reverse MK-801-induced deficits in social interaction and hyperactivity in Sprague-Dawley rats. J Psychopharmacol (Oxford). 2012;26(10):1317-1332.

36. Malone DT, Jongejan D, Taylor DA. Cannabidiol reverses the reduction in social interaction produced by low dose delta(9)-tetrahydrocannabinol in rats. Pharmacol Biochem Behav. 2009;93(2):91-96.

37. Peres FF, et al. Cannabidiol prevents motor and cognitive impairments induced by reserpine in rats. Front Pharmacol. 2016;7:343. 
38. Campos AC, Moreira FA, Gomes FV, Del Bel EA, Guimarães FS. Multiple mechanisms involved in the large-spectrum therapeutic potential of cannabidiol in psychiatric disorders. Philos Trans $R$ Soc Lond, B, Biol Sci. 2012;367(1607):3364-3378.

39. Guimarães FS, Chiaretti TM, Graeff FG, Zuardi AW. Antianxiety effect of cannabidiol in the elevated plus-maze. Psychopharmacology (Berl). 1990;100(4):558-559.

40. Kaplan JS, Stella N, Catterall WA, Westenbroek RE. Cannabidiol attenuates seizures and social deficits in a mouse model of Dravet syndrome. Proc Natl
Acad Sci USA. 2017;114(42):11229-11234.

41. Kim H, Lim CS, Kaang BK. Neuronal mechanisms and circuits underlying repetitive behaviors in mouse models of autism spectrum disorder. Behav Brain Funct. 2016;12(1):3.

42. Pelc K, Cheron G, Dan B. Behavior and neuropsychiatric manifestations in Angelman syndrome. Neuropsychiatr Dis Treat. 2008;4(3):577-584.

43. Roberto M, Madamba SG, Moore SD, Tallent MK, Siggins GR. Ethanol increases GABAergic transmission at both pre- and postsynaptic sites in rat central amygdala neurons. Proc Natl Acad
Sci USA. 2003;100(4):2053-2058.

44. Deiana S, et al. Plasma and brain pharmacokinetic profile of cannabidiol (CBD), cannabidivarine (CBDV), $\Delta^{9}$-tetrahydrocannabivarin (THCV) and cannabigerol (CBG) in rats and mice following oral and intraperitoneal administration and CBD action on obsessive-compulsive behaviour. Psychopharmacology (Berl). 2012;219(3):859-873.

45. Millar SA, Stone NL, Yates AS, O'Sullivan SE. A systematic review on the pharmacokinetics of cannabidiol in humans. Front Pharmacol. 2018;9:1365. 